\title{
Design and Implementation of an Application Layer Protocol for Reducing UDP Traffic Based on User Hints and Policies
}

\author{
William Kulju ${ }^{1}$ and Hanan Lutfiyya ${ }^{2}$ \\ 1 DB2 CLI/ODBC Application Enablement \\ IBM Toronto Laboratory \\ Toronto, Ontario, Canada \\ wkulju@ca.ibm.com \\ ${ }^{2}$ Department of Computer Science \\ The University of Western Ontario \\ London, Ontario, Canada N6A 5B7 \\ hanan@csd.uwo.ca
}

\begin{abstract}
This paper presents an application-layer protocol for UDP that makes use of user hints (e.g., a screen saver being invoked or covering a window with another) to reduce network traffic, which in turn may help reduce congestion. The architectural components used to implement the application-layer protocol can generally be applied to a reduction in resource (e.g., CPU) consumption. We present an architecture and experimental results.
\end{abstract}

Keywords. Policies, User hints, UDP, management

\section{Introduction}

Interactive applications (e.g., video conferencing) do not react well to TCP's congestion control mechanisms. As a result, many developers use UDP for multimedia applications. UDP provides little functionality beyond basic data transfer. Additional functionality is to be implemented at the application layer by the developer. This has the effect of the developer creating their own 'tailor-made' application-layer protocol with UDP as the underlying data transfer mechanism. These application-layer protocols have included congestion control schemes (e.g., [4). These protocols augment UDP to provide a particular class of multimedia applications (e.g., video conferencing tools, shared whiteboards) with services they would likely find useful (e.g., time-stamping for video applications).

One approach to reducing UDP traffic is based on the use of user hints. For example, if a video-on-demand client is running in a window that is minimized then this is a strong 'hint' that the user's interest has changed. If the source of the video source is made aware of this fact, it could reduce its output to audio feed, pause the video feed or slow down the rate that it sends the video feed. Basically, the source can alter its transmission behaviour in a fashion that

The original version of this chapter was revised: The copyright line was incorrect. This has been corrected. The Erratum to this chapter is available at DOI: $10.1007 / 978-3-540-45812-8 \_28$ 
minimises unwanted network traffic. These user actions are referred to as hints. Hints such as minimising and restoring windows, the covering and uncovering of windows, and the activation and deactivation of screen savers and locks are all excellent indicators of the interest users have in their applications.

This paper presents an application-layer protocol for UDP that makes use of hints. The design of the architectural components that are used to implement the protocol can also be used for not only reducing UDP traffic but also reducing consumption of host computing resources. The paper is organized as follows. Section 2 describes considerations to take into account when contemplating the design. Section 3 describes the architecture and the interactions among the components of the architecture. Section $\square$ describes the prototype implementation and initial experimental results. Section 5 describes related work. Section 6 has a detailed discussion that analyzes the protocol and the architecture. Section $\mathbf{Z}$ states the conclusions and some of the future work.

\section{Consideration in Design}

A protocol can be informally defined as a set of well-known rules and formats needed for communication between two processes. Protocols enforce policies. A policy specifies actions that are to be undertaken by a protocol end-point when an event occurs. For example, a TCP source re-transmits data packets that have not been acknowledged (an action) before a time-out period expires (an event). The action ensures data 'lost' in the network is re-transmitted, which is necessary to effect TCP's guaranteed data delivery. Similarly, a TCP source throttles its transmission rate by half (an action) when the same event described earlier occurs in order to resolve network congestion.

There are a number of application-layer protocols (used to augment UDP), each incorporating a congestion control scheme, that have been proposed for various types of multimedia applications Application-layer protocols used to augment UDP with a congestion control scheme (e.g., 4]) provide a particular class of multimedia applications (e.g., video conferencing tools, shared whiteboards) with services they would likely find useful (e.g., time-stamping for video applications). Therefore, a developer creating an application that fits into one of these classes could benefit by using the available protocol associated with that class rather than trying to make their own.

A shortcoming of these protocols is that the enforced policies for congestion control are static. In other words, once the protocol source code has been compiled, the congestion control algorithm of the resultant protocol executable is immutable. Thus, protocol designers usually decide on a single set of congestion control policies to be enforced. This often leads to a design of protocols based on worst-case requirements (e.g., 'computationally challenged' client and server machines connected by a 'slow' wireless link) since protocol developers cannot possibly anticipate in advance all the scenarios under which the protocol will be deployed. Therefore, developers are encouraged to design protocols that overcompensate rather than under-compensate [7] (e.g., a protocol might statically 
reserve more memory for input/output buffering than is realistically required as protection for the rare situations - perhaps when it is used on a 'slow' machine - where buffer overflow might otherwise occur). This often means that protocols do not always effectively manage resources.

The design of the application-layer protocol in this work allows for dynamic policies in the following sense: Policies can be dynamically negotiated between client(s) and server(s) both at the beginning of, and throughout a multimedia session. Once negotiated, these policies are then enforced for the duration of the ensuing session (or until they are re-negotiated) in a manner that is transparent to the applications relying on it.

\section{Architecture}

The management architecture is graphically depicted in Figure 1. The following example is used to help describe the architectural components. Assume that there is a video-on-demand session in progress between a client video application and a streaming server application connected by the Internet. We further assume that the client video display is presently a top-level window being viewed by an end-user. Now suppose that the end-user decides to check his e-mail. To do so, he opens a separate e-mail window. Let us also suppose he immediately positions this new window on his desktop in such a way that it covers the entire video display. This suggests that the end-user is less interested in the video display since he cannot even see it. A reasonable policy to enforce under these circumstances is the following: 'When the video display window area is at least $75 \%$ covered by other windows then the streaming video service provider should pause the stream of video from the server to the client. This video stream is continued when the video display window area is less covered with other windows'.

\subsection{End-User Interaction Monitors}

An end-user typically interacts with a video display window, keyboard, a mouse and a chair. These entities can be characterized by attributes that change in response to an end-user's behaviour. For example, a video display window has an attribute that represents its obscurity level (i.e., extent to which the end-user has covered the video display window with other windows). Another example is that of a workstation monitor. It has an attribute that represents whether or not the screen saver is activated as a result of end-user activity. When the value of these attributes changes, it may be a 'hint' that an end-user's interest in a multimedia application has also changed. Detecting 'hints' is the responsibility of end-user interaction monitors.

Each interaction monitor monitors an attribute within the client-side multimedia environment for changes in the value of that attribute. Upon detecting an attribute change, the monitor then determines if a particular condition on this attribute has become 'satisfied' or 'unsatisfied'. If so, the end-user interaction monitor notifies interested objects (e.g., client coordinator, which is discussed 


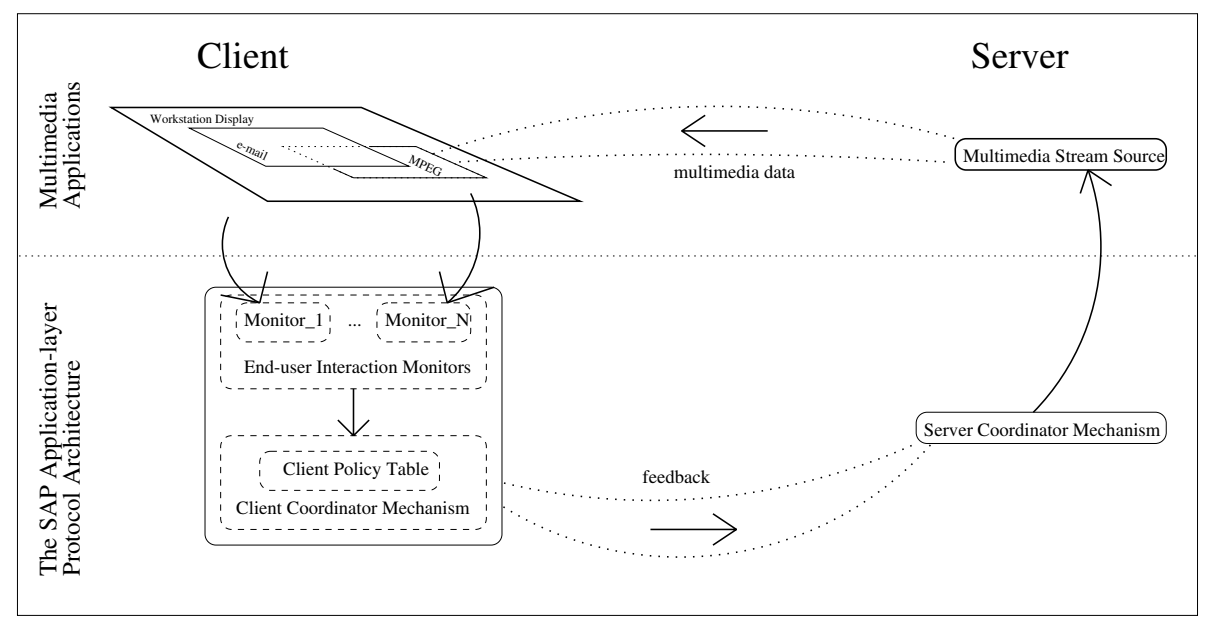

Fig. 1. The protocol used to (re)allocate streaming video server resources labelfig:client-server

in the next section) about the changed attribute condition. There may be more than one attribute condition that is evaluated.

\section{Definition 1.}

An attribute condition is defined as follows:

\section{attribute condition $=($ anAttribute, $($ comparisonOperator, aThreshold $))$}

where anAtribute denotes the attribute being monitored for value changes by the interaction monitor, aThreshold denotes a threshold that the value of anAttribute is being compared to, and comparisonOperator denotes the comparison operator by which the value of anAttribute and aThreshold are to be compared.

\section{Example 1.}

Suppose a 'window obscurity' monitor detects changes in the attribute representing how much of the video display window has been covered by other windows. Let $x$ denote this attribute. The monitor could evaluate the attribute condition $(x,(>=, 75))$ (i.e., "At least $75 \%$ of the total video display window area is covered by other windows") whenever a change in the value of $x$ is detected.

An end-user interaction monitor maintains a list of attribute conditions and provides methods that includes initializing and creating an attribute condition, changing parameters in an attribute condition, and allowing an external object to register with the end-user interaction monitor to receive notification when a previously 'unsatisfied' attribute condition has become 'satisfied' and when a previously 'satisfied' attribute condition has become 'unsatisfied'. 


\subsection{Client Coordinator}

The client coordinator maintains a table that maps attribute conditions to actions. This table is referred to as the client policy table and it represents the policies to be enforced. Whenever an end-user interaction monitor determines that a previously 'true' attribute condition has become false (or vice versa), it notifies the client coordinator. The client coordinator updates the client policy table that a particular attribute condition has changed from unsatisfied to satisfied or from satisfied to unsatisfied. Based on this information, it is then determined what actions the client coordinator should execute.

\section{Definition 2.}

A policy is defined as follows 1

$$
\text { policy } \left.=\begin{array}{c}
\left(\text { attributeConditon }_{1}, \ldots, \text { attributeCondition }_{\mathrm{n}}, \text { actionList }_{1},\right. \\
\text { actionList }_{2}
\end{array}\right)
$$

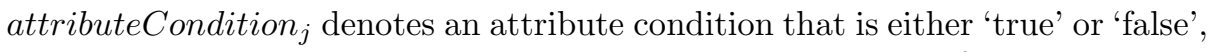
action List $_{1}$ denotes actions the client coordinator is to execute if the conjunction of attributeCondition through attributeCondition $_{n}$ has just become 'true' (i.e., it has gone from 'unsatisfied' to 'satisfied'). In contrast, action List $_{2}$ denotes actions the client coordinator is to execute if the conjunction of attributeCondition ${ }_{1}$ through attributeCondition $n$ has just become 'false' (i.e., it has gone from 'satisfied' to 'unsatisfied').

An action that is found in either actionList or $_{\text {actionList }}$ of the policy definition is defined as follows:

\section{Definition 3.}

An action is defined as follows:

$$
\text { action }=(\text { targetObject, (actionMethod, actionMethodParameter }))
$$

targetObject denotes an object and actionMethod denotes a method to be executed on targetObject. targetObject may be either local to or remote from the client multimedia application process space. Finally, actionMethodParameter denotes zero or more parameters to be included with the actionMethod call (e.g., a command message, another action). Note that actionMethodParameter is optional in any particular action.

\section{Example 2.}

Suppose a streaming video service provider would like to 'pause' all transmitted bandwidth to a client video application end-user whenever more than $75 \%$ of the total video display play window area is covered by other windows.

(("percentage of the total video display window area covered by other windows",

1 This definition of policy is based on that found in IETF standards at http://www.ietf.org. 


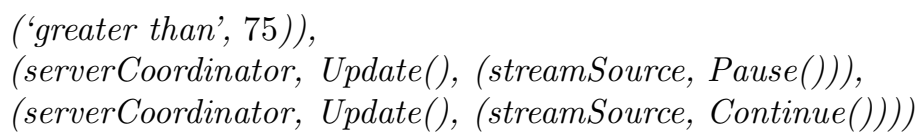
This policy can be interpreted as follows: When the video display window area is $75 \%$ covered by other windows then the streaming video service provider should pause the stream of video from the server to the client. This video stream is continued when the video display window area is less covered with other windows.

\subsection{Server Coordinator}

The server coordinator is a server-side mechanism that performs two tasks. The first of these tasks results in providing entries for the client policy table. When the client initiates a multimedia session, the client coordinator sends the server coordinator a list of attributes that is referred to as the attribute profile. This list specifies all client-side attributes the client coordinator is able to have monitored for value changes by end-user interaction monitors. When the server coordinator receives the attribute profile, it examines its list of policies. If the attribute condition of the policy has a corresponding match in the attribute profile, the server coordinator places that policy on a list that is sent to the client coordinator. Each policy on the list is added to the client coordinator's policy table.

The second task is that of dynamic adjustment of the service transmitted to a client in response to feedback from the client. Feedback is information is provided by the client coordinator. The feedback is used by the server coordinator to adjust the quality of the multimedia stream transmitted to the client application associated with that particular client coordinator.

The server coordinator maintains a list of handles to client coordinators. Furthermore, the server coordinator is able to differentiate between client coordinators via these handles, thus implying that different dynamic adjustments to the quality of the multimedia stream can be done based on the client.

\subsection{Interactions}

This section describes the relationship between the management and application components.

The architecture assumes that the client coordinator is implemented as a thread of the process executing the application. This thread is initiated through instrumentation code (code which is inserted into an application at strategic locations). Each application has one client coordinator. A similar assumption is made for the server coordinator. It sometimes makes sense for an end-user interaction monitor to interact with more than one client coordinator (e.g., an end-user interaction monitor that detects the presence or absence of a screen saver). End-user interaction monitors provide a set of methods. One of these 
methods includes a registration method that allows client coordinators to receive notification when a previously 'unsatisfied' attribute condition has become 'satisfied' (or vice-versa). The registration method allows for the specification of the attribute condition (i.e., the comparison operator and a threshold to compare values of the attribute). Thus, it is possible for an end-user interaction monitor to maintain a list of entries where each entry is an attribute condition and handles for client coordinators. When the value of the attribute changes then each attribute condition is evaluated. If there is a change from 'satisfied' to 'unsatisfied' (or vice-versa) then a notification is sent to the client coordinators that registered to receive a notification when the result of an attribute condition evaluation changes.

As described earlier, the server coordinator receives a list called the attribute profile from the client coordinator. This list specifies all client-side attributes that the client coordinator is able to have monitor through end-user interaction monitors. The client coordinator creates this list from its profile file or from a profile list received from a management agent. The profile file(list) contains entries that are a pair, with the first element being the attribute name and the second element being the location of a client-side executable or dynamic link library to an interaction monitor that monitors the attribute. It should be noted that when the client coordinator is initialised it is given the handle of a server coordinator.

Upon receiving an attribute profile from a client coordinator, the server coordinator examines its server policy list. Each entry of this list is a policy. Upon receiving an attribute profile from a client coordinator, the server coordinator examines each attribute condition of each policy in the server policy list. If the attribute component of the attribute condition of a policy can be found in the attribute profile of the client coordinator, then the server coordinator places that policy in a list. This list is sent to the client coordinator. The client coordinator uses this to add entries to the client policy table and instantiates end-user interaction monitors as needed.

This allows for a server policy list to be created in advance that specifies an arbitrarily large number of policies and attribute conditions within those policies. Moreover, it can be assumed that any particular client coordinator will only be able to have an arbitrary fraction of the attribute conditions in the list monitored by end-user interaction monitors. The server coordinator dynamically chooses the policies it wants an arbitrary client coordinator to enforce in a multimedia session, given the capabilities of that client coordinator.

This approach also allows a server policy list to provide differentiated services by providing different policies for different client processes.

Policies can be re-negotiated as needed during an ensuing session. Examples of why this may be necessary include a change in the number of multimedia session participants, changed resource conditions at the client or server, or changes in congestion within the network. This is the result of the client coordinator being designed so that it provides methods that allow for the policies of the client policy table to be modified. If the change in policy involves a change in the 
threshold value used in an attribute condition, then the method of the end-user interaction monitor that changes the threshold is called by the client coordinator.

\section{Implementation}

This section describes the implementation of a prototype based on the architecture described in the previous section.

\subsection{Client and Server Coordinators, Policy Table and Interaction Monitors}

The client coordinator, server coordinator and policy table were implemented as objects in Java 2.0 [1] Remote communication between the client coordinator and server coordinator objects is achieved through use of the Java Media Framework 2.0 (JMF) version of the Real-time Transfer Protocol (RTP) 1]. RTCP (Realtime Transfer Control Protocol) 122 is used for client and server coordinator communication.

We implemented the following end-user interaction monitors for incorporation within our prototype. The window obscurity monitor detects when a specified window on the end-user's monitor has become obscured after being unobscured (based on some threshold value) or has become unobscured after being obscured. It was implemented using X11 [8]. The second interaction monitor implemented is a video frame rate monitor that detects when the rate of video frames rendered to the end-user's workstation screen has fallen below a certain threshold rate for a specified duration. The third interaction monitor detects when the screen saver has been activated.

The attribute profile (which is sent to the server coordinator) of the client and the server's list of policies are found in files.

\subsection{Applications and Policies Used in Experimentation}

We constructed a video receiver application and modified an existing streaming video server to use our protocol. The video receiver application was written entirely in Java using Java 1.2 and JMF 1.2. Video data transmitted by a remote streaming video server is forwarded to the receiver by an RTP channel. In JMF, the local RTP end-point within the client that receives this data is referred to as the client's 'session manager'. In JMF, the local RTP end-point within the client that receives this data is referred to as the client's 'session manager'. A session manager also maintains a RTCP channel for communication of application-specific control information between end-points. End-points communicate via RTCP. This allows us to leverage the channel already made available by the session manager rather than implement a second RTCP (and hence a second RTP) channel. The server application is a modified version of the JMStudio multimedia application. The policies used are informally stated 
as follows: (1) If the video display is obscured by $75 \%$ or more, the server coordinator is to pause transmission to the client. This was more formally stated in Example 2. (2) If the screen saver is on, the server coordinator is to pause transmission to the client.

\subsection{Testing and Experiments}

We will now briefly summarize some of the experimental results. The following discussion is based on the first policy, but similar results were achieved for the other policy. After the policy agreed upon by the client and the server coordinators, we took measurements from our testing tools. The UNIX 'top' utility on the server machine revealed that roughly $45 \%$ of server machine CPU utilisation was devoted to transmitting the video data and that 39 megabytes of RAM was reserved by the source application. Furthermore, the cumulative number of packets sent by the server was increasing steadily. These observations were largely identical to those measured with the unmodified JMStudio video server. Similarly, a 'top' utility on the client machine revealed that approximately $7 \%$ of client machine CPU effort was devoted to decoding and rendering the received video data, while 32 megabytes of RAM was reserved by the receiver application. These observations closely paralleled those made with an otherwise equivalent receiver application that did not use our prototype. This suggests that our protocol does not have much overhead.

When portions of the 'actual' video display window were obscured such that the total was less than $75 \%$ of the total video display window area, there was no visible reaction. However, once more than $75 \%$ of the total video display window area had become obscured, video transmission was paused at the source within at most 5 seconds (in all of our trials). Then, almost instantly, the rendering of video to the client workstation monitor halted as well. In this state, the CPU utilisation of both source and receiver applications quickly fell to $0 \%$ (although reserved RAM remained unchanged on both client and server machines). The packets sent also fell to zero. These results suggest that our prototype had significantly reduced the amount of 'unwanted' data traffic entering the network and that it was highly reactive since full traffic reduction was achieved within seconds of the source video data becoming 'unwanted'.

Generally, the prototype was extensively tested under different circumstances including the following: (i) A client may have no interaction monitors, which basically implies that no policies may apply and hence there is no opportunity to reduce resource consumption; (ii) The client's attribute profile has attributes that do not match with any policies that the server has; (iii) The server policy file may be empty. In all cases, the prototype worked.

\section{Related Work}

The work closest to ours is that found in [4] which describes a dynamic video conferencing model called SCUBA. SCUBA is a scalable control protocol that 
employs 'hints' about end-user interest that occur at the workstation display to adapt the transmission rate of media sources to the receivers that are part of the multicast session. Our work differs in that it provides more flexibility in the types and number of user hints (SCUBA only takes into account window placement). We provision for incorporating additional end-user hints. Second, the SCUBA policies are hardcoded. A change requires re-compilation.

There are a number of other works that address network congestion control. Receiver-driven protocols (e.g., [10]) are where receivers are given control over the transmission behaviour of sources by the protocol. Sender-driven protocols (e.g., [5]) are where senders determine their own bandwidth transmission rate (usually on the basis of receiver feedback). Receivers may be best equipped to make decisions regarding issues local to them (e.g., network congestion). But in a multicast session, it is generally not a good idea to let individual receivers adjust source transmission behaviour since the receiver will likely have no regard for the effects of these changes on other receivers. Hence the logic behind sender-driven protocols is to have receivers submit feedback to the source so that the source can then make decisions that will best benefit the entire group.

In both types of protocols there is a notion of policies, but the policies are static. Thus, the congestion control achieved through these policies can be ineffective when used under the precise conditions for which the protocol was defined.

There has been relatively little research (e.g., [39 13]) and industrial effort investigating how to effectively make use of hints of user activity to guide resource management. The work is domain-specific and limited in scope. While these issues were raised in [3], no design or implementation work was ever completed. [9] also examines these issues, but only in the context of their own 3D landscape environment, and not generally available production environments. From industrial research, Microsoft Windows 95, 98, and NT [11] can capture some user hints to adjust application priorities and time quanta slightly as applications are foregrounded and backgrounded. Some flavours of UNIX have limited support for user hints, by adjusting scheduling according to I/O activity.

\section{Discussion}

This section describes the requirements that the proposed protocol satisfies and provides a broader context for this work.

Use of Multiple Interaction Monitors. It is possible for our protocol to receive input from a potentially arbitrary set of end-user interaction monitors. In our implementation client coordinators have access to a file (i.e., the attribute profile) that specifies both the available end-user interaction monitors and details on how to instantiate each of those monitors. The addition of new end-user interaction monitors means an additional entry in the policy file for the client. If the server specifies polices in its policy file that have attributes in the attribute condition that cannot be monitored by the client then that policy is not applied 
to the client. This allows the server process to enforce policies for different client processes even though the different client processes monitor different attributes. Development Issues. On the surface, it would seem that the development of client and server coordinators as well as monitors is difficult and time-consuming. It should be noted that the client and server coordinators were designed to be independent of the specific applications. It should be noted that Definitions 1 , 2 and 3 can easily map to Java classes (as well as $\mathrm{C}++$ classes). The methods defined are relatively simple in that they are primarily being used to manipulate the structures found in Definitions 1, 2 and 3. We found that these classes could be applied to any of the policies and applications used. The coordinators are implemented as Java classes and can be reused by different applications.

The interaction monitors are more difficult. The 'window obscurity' monitor is rather difficult to implement, but it can be used by multiple applications. It should be noted that there would have to be a 'window obscurity' monitor class for each platform. The monitoring of the frame rate can be encapsulated as a class and used by multiple applications. It is this reuse aspect that reduces the amount of development work.

The window obscurity and frame rate monitors execute outside of an application. However, the frame rate monitor are part of an application's instrumentation. The application developer needs to know the methods of the class encapsulating the monitoring, but is not concerned with the details on how the frame rate is actually calculated.

Flexibility. Example 2 states that if the video display window area is $75 \%$ covered by other windows then the streaming video service provider should pause the stream of video from the server to the client. However, it may be desirable to pause the video but not the audio. This can be done by having a policy that states that the action to be taken is to only pause the video.

Specification and Distribution of Policies. There already exists formalisms for specifying policies. One example can be found in [6]. Our policies can easily be specified using this formalism.

Dynamically Changing Policies. Assume that it is decided that a server process changes the policy about window obscurity. For example, the server is to be notified when video display window area is $60 \%$ covered as opposed to $75 \%$ covered. The server coordinator process informs the client coordinator, which then notifies the end-user interaction monitor to change the threshold associated with the attribute condition and the specific client coordinator.

\section{Conclusions and Future Work}

The main significance of our work is that it permits both the monitors that detect end-user activity, and the policies specifying actions to undertake when that activity occurs, to be dynamically negotiated between client(s) and server(s) at run-time. This is an important consideration because receivers in a multimedia session might not all have the same capacity for detecting end-user activity or responding to that activity. For example, detecting whether or not an application 
end-user is seated in the chair before their workstation first requires some sort of sensory device especially geared to the task. If a particular receiver does not possess this device, then our work permits the source to decide upon an alternative action (e.g., substitution of hints from the 'seat monitor' with hints detected by another sensory device).

Our work allows for dynamic modification of policies. As discussed in the previous section, it may be decided that a server is to be notified when the video display window area is $60 \%$ covered as opposed to $75 \%$ covered. This may be done if high network congestion is detected. This change permits the server to reduce the number of packets it is sending based on user interest. Thus, our approach permits congestion control policies to be finely tuned at run-time so that they are as aggressive as possible.

There are a number of issues to be addressed in the future and are briefly described as follows.

- This work relies on the assumption that the attribute profile and the server policy list have an agreement on attribute names. Thus, two syntactically identical attribute names are assumed to be semantically equal. We will closely examine how to ensure this assumption. We will examine the use of XML to specify universal attribute semantics, but let the client and server coordinators apply their own syntax to those semantics. This can be accomplished by having end-user interaction monitor developers associate an external document type definition (DTD) with each new interaction monitor release. That DTD would specify the semantics of the attribute condition that monitor was designed to detect. All DTDs constructed could be deposited in special Internet DTD repositories, which would make them 'universal'. A server coordinator uses the DTD to reconstruct the attribute in a syntax the server coordinator understands. This facilitates the use of user hints and policies for application components in different administrative domains.

- We are especially interested in applying our work to a production environment involving machines of faculty, staff and graduate students in our department as well as using at least one application such as video-conferencing for further validation. We will install the prototype software on these machines (of course, with permission) and evaluate different policies.

- We will apply our protocol to SCUBA and other protocols (both send and receiver oriented) and evaluate their performance.

- Policies may conflict. To make this protocol truly successful, we must find a heuristic for detecting this type of conflict.

\section{Copyright Information}

Java and all Java-based trademarks are trademarks of Sun Microsystems, Inc. in the United States, other countries, or both. Microsoft, Windows, Windows NT, and the Windows logo are trademarks of Microsoft Corporation in the United 
States, other countries, or both. UNIX is a registered trademark of The Open Group in the United States and other countries. Other company, product, or service names may be trademarks or service marks of others.

\section{Acknowledgements}

This work is supported by the National Sciences and Engineering Research Council (NSERC) of Canada, and the Canadian Institute of Telecommunications Research (CITR).

\section{References}

1. Sun Microsystems Inc. Internet site, www.java.sun.com/products. 270] 270]

2. Java Media Framework API Guide. Sun Microsystems Inc, JMF 2.0 FCS, November 1999. 270.

3. M. Alfano. Design and Implementation of a Cooperative Multimedia Environment with QoS Control. Computer Communications (21), Elsevier Science, Fall 1997. 272. [272]

4. E. Amir, S. McCanne, and R. Katz. Receiver-driven bandwidth adaptation for light-weight sessions. ACM Multimedia, November 1997. [263, [264] [271]

5. M. Barcellos and P. Ezhilchelvan. An end-to-end reliable multicast protocol using polling for scaleability. Proceedings of the Conference on Computer Communications (IEEE Infocom), (San Francisco, California), page 1180, March 1998. [272]

6. N. Damianou, N. Dalay, E. Lupu, and M. Sloman. Ponder: A language for specifying security and management policies for distributed systems: The language specification (version 2.1). Technical Report Imperial College Research Report DOC 2000/01, Imperial College of Science, Technology and Medicine, London, England, April 2000. 273

7. S. Floyd. Internet research: Comments on formulating the problem. an open letter prompting discussion. Unpublished paper, January 1998. www.aciri.org/floyd/. 264

8. A. Nye. Xlib Programming Manual for Version 1.1. O'reilly \& Associates Inc., 1995. 270

9. M. Ott, G. Michelitsch, D. Reininger, and G. Welling. An Architecture for Adaptive QoS and its Application to Multimedia Systems Design. Computer Communications Special Issue on Guiding Quality of Service into Distributed Systems, August 1997. 272, 272

10. S. Raman and S. McCanne. Scaleable data naming for application level framing in reliable multicast. In ACM Multimedia '98 (Bristol, England), September 1998. 272

11. J. Richter. Advanced Windows, Third Edition. Microsoft Press, 1997. [272

12. H. Schulzrinne. RFC 1889, RTP: A Transport Protocol for Real-Time Applications. November 1995. Available from: www.cs.columbia.edu/ hgs/rtp/. [270]

13. J. Walpole, R. Koster, S. Cen, C. Cowan, D. Maier, D. McNamee, C. Pu, D. Steere, and L. Yu. A Player for Adaptive MPEG Video Streaming Over The Internet. Proceedings of the 26th Applied Imagery Pattern Recognition Workshop, Washington DC, October 1997. 272] 